\title{
Implicações da cultura no cuidado da equipe de saúde da família em uma comunidade quilombola
}

\author{
Implications of culture for care by a family health team in a quilombola community \\ Implicaciones de la cultura en el cuidado del equipo de la salud de la familia en una \\ comunidad quilombola
}

Daniela Bastos Silveira ${ }^{I}$ Maria Delma Freitas das Chagas ${ }^{I I}$; Thaisa Santos da Hora ${ }^{I I I}$; Donizete Vago Daher ${ }^{I V}$; Sonia AcioliV

\begin{abstract}
RESUMO: O objeto deste estudo é a cultura e suas implicações no cuidado da equipe de saúde da família em uma comunidade quilombola. Objetivou-se analisar aspectos da cultura afrodescendente intervenientes no cuidado à saúde da comunidade quilombola, na visão da equipe de saúde da família. Estudo descritivo-exploratório com abordagem qualitativa. Teve como cenário a comunidade quilombola de Machadinha, em Quissamã - RJ, e como sujeitos, sete profissionais. A coleta de dados foi realizada mediante grupo focal e observação não participante. Os dados foram submetidos à análise temática de Bardin, em julho de 2014. Como resultado, identificou-se que os hábitos culturais dessa população estão, ainda, fortemente presentes, sobretudo na culinária, na dança e no uso de ervas medicinais. Tais hábitos exigem dos profissionais um diálogo entre saber técnico e saber popular. Concluiu-se que a equipe de saúde da família precisa considerar sua prática observando a cultura local como norteadora no processo de cuidar. Palavras-Chave: Estratégia de saúde da família; enfermagem; cultura; população quilombola.
\end{abstract}

ABSTRACT: The object of study was culture and its implications for the family health care team in a quilombola community. This exploratory, qualitative, descriptive study examined aspects of Afro-descendant culture that influence quilombola community care from the family health care team's viewpoint. The scenario was the Machadinha quilombola community at Quissamã, Rio de Janeiro State, and seven health personnel were the subjects. Data were collected through focus group and non-participant observation in July 2014, and analyzed using Bardin thematic analysis. The population's cultural habits were found still to be strongly present, especially in cooking, dancing, and use of medicinal herbs. Those habits require that health personnel dialogue between technical and traditional knowledge. It was concluded that the family health team needs to consider its practice in view of local culture as a guide for the care process.

Keywords: Family health strategy; nursing; culture; quilombola population.

\begin{abstract}
RESUMEN: El objeto de este estudio es la cultura y sus implicaciones en el cuidado del equipo de salud de la familia en una comunidad quilombola. Se buscó analizar aspectos de la cultura afrodescendiente que intervienen en el cuidado a la salud de la comunidad quilombola, desde la visión del equipo de la salud de la familia. Es un estudio descriptivo-exploratorio de abordaje cualitativo. El escenario se constituyó de una comunidad Quilombola de Machadinha ubicada en Quissamã, Estado de Rio de Janeiro. Los individuos analizados fueron siete profesionales. La recolección de datos fue realizada mediante grupo focal y observación no participante. Los datos fueron sometidos al análisis temático de Bardín, en julio de 2014. Como resultado, se ha verificado que los hábitos culturales de esa población siguen, todavía, fuertemente presentes, sobre todo en la culinaria, en la danza y en el uso de hierbas medicinales. Dichos hábitos exigen de los profesionales un diálogo entre saber técnico y saber popular. Se concluye que el equipo de salud de la familia necesita considerar su práctica observando la cultura local como norte en el proceso de atención. Palabras Clave: Estrategia de salud de familia: enfermería: cultura: población quilombola.
\end{abstract}

\section{INTRODUÇÃO}

A cultura é compreendida como uma composição de elementos que caracterizam quaisquer atividades que não sejam demarcadas pela biologia, sendo compartilhadas por diferentes integrantes de um grupo social. Envolvem questões como valores, símbolos, práticas, tradições e costumes. É um produto coletivo da vida humana. São elementos a partir dos quais os sujeitos constroem significados para as ações e interações sociais concretas e, dessa forma, resistem às configurações sociais definidas ${ }^{1}$

Por meio da cultura, expressam-se as mais diversas características da realidade dos povos, nações e grupos

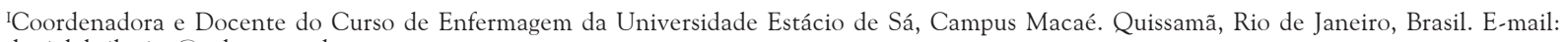
danielabsilveira@yahoo.com.br.

"Enfermeira formada pela Universidade Estácio de Sá. Quissamã, Rio de Janeiro, Brasil. E-mail: mdelmafisio@hotmail.com.

IIIEnfermeira formada pela Universidade Estácio de Sá. Macaé, Rio de Janeiro, Brasil. E-mail: thaisabarcelos@hotmail.com.

IV Professora Associada do Departamento de Enfermagem Médico-Cirúrgica da Universidade Federal Fluminense. Niterói, Rio de Janeiro, Brasil. E-mail: donizete@predialnet.com.br.

vDoutora em Saúde Pública. Professora Adjunta do Departamento de Saúde Pública da Faculdade de Enfermagem da Universidade do Estado do Rio de Janeiro, Brasil. E-mail: soacioli@gmail.com. 
humanos, como na dança, na arte, na culinária e nos diferentes modos de viver ${ }^{2}$

A aproximação do trabalhador em saúde com a cultura de uma população possibilita que o cuidado prestado por ele ganhe valor e sentido, pois dito profissional pode compreender a forma como as pessoas sentem e vivem a sua saúde.

Conforme estabelecido pela Política Nacional de Atenção Básica ${ }^{3}$, a Estratégia de Saúde da Família (ESF) deve nortear seu trabalho considerando o sujeito em sua singularidade e inserção sociocultural, com vistas à garantia de uma assistência integral. A saúde da família destaca-se, assim, como uma estratégia de reorganização dos serviços de saúde a partir da substituição de um modelo de saúde, com um olhar focado apenas no indivíduo e em seu corpo fragmentado, para ampliá-lo, considerando seu contexto familiar e seus valores socioculturais.

Este artigo é desdobramento de uma pesquisa mais ampla que analisa a implicação da cultura em práticas de cuidado de enfermagem. A cultura e suas implicações no cuidado de enfermagem da equipe de saúde da família em uma comunidade quilombola é o objeto deste estudo. A partir do contexto descrito foram estabelecidas como questões norteadoras: Quais são os hábitos culturais remanescentes da cultura afrodescendente da comunidade quilombola de Machadinha? Como o contexto cultural da comunidade pode subsidiar ações de promoção e prevenção da saúde a partir do trabalho da equipe de saúde da família?

Com base nas questões norteadoras, foi selecionado como objetivo analisar aspectos da cultura afrodescendente intervenientes no cuidado à saúde da Comunidade Quilombola de Machadinha (Quissamã-Rio de Janeiro), a partir da visão da equipe de saúde da família.

\section{ReFerencial TeóRICO}

O estudo teve como embasamento teórico a Política Nacional de Atenção Básica (PNAB) ${ }^{3}$, a Política Nacional de Saúde Integral da População Negra (PNSIPN) ${ }^{4}$, Educação Popular, ${ }^{5,6}$ a Teoria da Diversidade e Universalidade do Cuidado Cultural de Madeleine Leninger ${ }^{7} \mathrm{e}$ documentos que desenham a história de Machadinha ${ }^{8,9}$.

A PNSIPN publicou que, de acordo com o censo demográfico de 2000, o Brasil possui 54\% de habitantes que se consideram brancos, $45 \%$ negros ou pardos e $0,4 \%$ indígenas ou amarelos ${ }^{4}$. Esses dados retratam a importância de um olhar mais cuidadoso à população negra, que possui características e necessidades próprias que merecem atenção especial do setor saúde e também da educação.

Dados como pobreza e nível de renda aparecem significativamente piores em relação à população branca. Pode-se, ainda, mencionar indicadores de saúde importantes como gravidez na adolescência, em que a população negra está quase 2\% maior que na população branca; além de maior risco de crianças menores de cinco anos morrerem por doenças infectoparasitárias ou por desnutrição. Quanto aos adultos, foi constatado que a taxa de morbimortalidade de negros é superior a dos branco ${ }^{4}$

É responsabilidade da ESF gerir as demandas de saúde de maior frequência do seu território, considerando os critérios de risco, a dinamicidade e as características próprias de uma determinada população ou grupo ${ }^{3}$. Nesse sentido, refletir sobre os aspectos singulares de uma população quilombola torna-se imprescindível para um cuidado singular, de qualidade e resolutivo pela equipe de saúde da família.

A Comunidade Quilombola de Machadinha apresenta, como principais características, a preservação das danças típicas dos escravos (jongo e fado) e da culinária rica em raízes, hábitos remanescentes do período da escravidão. Práticas como andar descalços e a utilização de ervas medicinais são muito comuns entre a população ${ }^{8}$.

Considerando que tanto a dança como a culinária podem estar ligadas às questões de saúde, há que se refletir acerca da utilização dos recursos culturais da população quilombola pela equipe de saúde da família, pois podem contribuir para reavivar tradições funcionando, também, como motivadoras para a realização de práticas de atividades físicas, com melhora da autoestima. Tais recursos são fundamentais no processo de construção e fortalecimento do vínculo entre a equipe e a população, bem como na construção conjunta do plano de cuidados prestados a esse grupo, de modo a valorizar o saber científico e o saber popular. Nessa direção, a cultura funciona como ferramenta para a prática da educação popular.

A educação popular e (em) saúde é um movimento social de uma classe de profissionais de nível técnico e pesquisadores comprometidos em interligar o diálogo entre o conhecimento técnico-científico e o conhecimento adquirido por meio das experiências e lutas da população pela saúde ${ }^{5,6}$. É a partir dessa concepção que as práticas de saúde da ESF no cuidado à população quilombola precisam se sustentar.

Referente à enfermagem, as práticas educativas configuram um elemento que compõe o processo de trabalho em saúde e se constitui como arcabouço do seu trabalho. Desse modo, o enfermeiro-educador na saúde coletiva exerce papel fundamental no desenvolvimento dessas atividades nos mais diversos cenários ocupados pela população ${ }^{10}$.

De maneira a nortear a sua prática considerando as questões culturais, o enfermeiro pode balizar suas ações apoiado na Teoria Transcultural de Madeleine Leininger. A teórica considera que a percepção de mundo dos indivíduos na condição social e cultural afeta seu estado de saúde, doença e bem-estar ${ }^{7}$.

Concebe-se cultura como os valores, crenças, normas e modos de vida aplicados, que foram internalizados, compartilhados e disseminados por determi- 
nados grupos, gerando pensamentos, decisões e ações de forma padronizada ${ }^{7}$. Assim, ao explorar o contexto cultural e aplicar esses conhecimentos como ferramenta para analisar as ações e decisões, o enfermeiro será capaz de prestar um cuidado diferenciado ${ }^{11}$.

\section{Metodologia}

Trata-se de um estudo descritivo-exploratório com abordagem qualitativa. $\bigcirc$ cenário compreendeu a Comunidade Quilombola de Machadinha, localizada em Quissamã, no Norte Fluminense do Estado do Rio de Janeiro. A coleta de dados foi realizada por meio da técnica de grupo focal e observação não participante em julho de 2014. A entrevista coletiva foi orientada por um questionário, contendo sete questões abertas, pré-testadas em uma unidade, com características semelhantes às de Machadinha. Após as entrevistas, houve sua transcrição na íntegra. Para identificação das falas dos sujeitos, optou-se pela utilização da letra inicial de seus nomes.

Os sujeitos foram sete profissionais de um total de 11 da equipe de ESF da unidade de Machadinha. Desse total, no momento da realização do grupo, havia um funcionário de férias, dois de folga e um presente que não aceitou participar. Estes entraram no critério de exclusão do estudo, enquanto o critério para inclusão foi que os profissionais tivessem pelo menos um ano de atuação nessa unidade e desejassem participar da pesquisa.

O tratamento de dados foi realizado a partir da técnica da análise de conteúdo de Bardin. Ela prevê o exame das comunicações com o intuito de compreender o conteúdo das mensagens ${ }^{12}$. Inicialmente, foi realizada a escuta das gravações para a organização das ideias, seguida da transcrição das falas e de sua respectiva interpretação. A seguir, foram delimitados temas que possuíam relação com os objetivos, seguidos da contagem e interpretação dos conteúdos para a construção das categorias que a posteriori foram examinadas.

Acerca dos aspectos éticos, o estudo atendeu ao disposto na Resolução n ${ }^{\circ} 466 / 12$. Os participantes assinaram o termo de consentimento livre e esclarecido, cientes de que poderiam retirar seu consentimento em qualquer fase da investigação. $\mathrm{O}$ estudo foi autorizado pela Prefeitura Municipal de Quissamã e aprovado por um Comitê de Ética e Pesquisa da área de saúde.

\section{Resultados e Discussão}

Na sistematização da análise, detectaram-se 80 temas de relevância abordados pelos integrantes da equipe de saúde, compostos a partir de unidades de registros que originaram duas categorias: Características culturais da comunidade de Machadinha remanescentes das suas origens afrodescendentes; e Estratégias utilizadas pela equipe de saúde da família para acompanhamento dos usuários.

\section{$1^{a}$ Categoria: Características culturais da comunidade de Machadinha remanescentes das suas origens afrodescendentes}

Observa-se que a população quilombola ainda preserva muitos hábitos e costumes da época da escravidão $^{8,9}$. Na culinária, identificou-se um grande consumo de tubérculos, carne e feijão, conforme observado nos depoimentos descritos:

Presença de uma alimentação forte à base de carne, farináceos, raízes e tubérculos. (A)

Foi a primeira vez que comi uma feijoada de peixe, sopa de leite, capitão, que é o bolinho de feijão com bacon, couve. (B)

Por meio dos depoimentos, observou-se que a equipe reconhece que a comunidade tem suas características próprias e procura preservá-las ${ }^{5-7}$. Nesse sentido, o conhecimento a ser construído sobre a saúde desse grupo precisa levar em conta sua dinâmica, além de incorporar o seu saber a partir de sua vivência. Os profissionais percebem que se trata de uma cultura alimentar aprendida por gerações anteriores, repassada por tradição oral, ao longo dos tempos ${ }^{1,2}$. Desse modo, é considerando os hábitos culturais dessa população que os trabalhadores em saúde podem perceber o sentido desta prática para o grupo, para, então, construir e compartilhar saberes.

A equipe compreende essa prática alimentar como característica positiva no sentido de manter as tradições e incentiva a formação e manutenção de hábitos saudáveis, como a criação de animais e plantação de hortas domiciliares, o que facilita o acesso a alimentos essenciais e ainda encoraja a preservação cultural do grupo ${ }^{7}$. Como ponto considerado a ser mais bem trabalhado, acerca da culinária local, a equipe coloca que há exagero no consumo de alguns alimentos, como os tubérculos, o que gera preocupação com o aumento de probabilidade de doenças que esses excessos acarretam.

Essa questão está identificada nas falas em que os profissionais mencionam essa preocupação.

[...] convencer um diabético aqui a diminuir esses carboidratos é complicado, não é fácil, mas também a gente não desiste. (D)

Diante disso, parece que os profissionais, apesar de respeitarem e valorizarem os hábitos alimentares dessa população, também realizam ações educativas no intuito de reorientar hábitos e incentivar novos. Nesse sentido, há de se considerar a necessidade de a equipe refletir sobre o cuidado ao realizar ações muito prescritivas e, talvez, até autoritárias com vistas à mudança de comportamento, pois é preciso considerar os sujeitos como protagonistas nesse processo. A análise aponta para um caminho no qual a equipe faz um esforço de valorizar tanto o saber científico quanto o popular, buscando a complementaridade entre ambos ${ }^{5,6}$. 
As questões da dança do jongo e das músicas tradicionais dos escravos surgem como expressões dos hábitos dessa população. O jongo é uma dança que relembra cantos de alforria e são executados em sincronia com as batidas de tambores 9 . Essa dança aparece como um hábito cultural valorizado pelos profissionais, conforme verificado no seguinte depoimento:

Essa questão da dança do jongo [...] é uma forma de expressão muito característica daquele lugar. [...] Outra questão que diferencia muito é a questão da cultura, a dança eu acho que é uma coisa muito importante para eles, é a maneira que eles têm desde o século retrasado de se comunicarem através dos tambores [...]. (B)

$O$ resgate da dança em Machadinha contribuiu para que as adolescentes fossem incentivadas a se inserirem no jongo. $\mathrm{O}$ depoimento a seguir é de um profissional da unidade, descendente de escravos. Ele sinaliza a importância da preservação das tradições e de sua transmissão às novas gerações ${ }^{7}$, de modo a manter a cultura viva, em movimento, promovendo a inserção das crianças e adolescentes nessas práticas e ensinando-as o valor dessa herança.

[...] hoje estamos iniciando o jongo com os netos e bisnetos [...]. (M)

Outro ponto importante, emergente das falas, é que a partir do momento da inserção dos adolescentes no jongo, houve uma redução significativa do índice de gravidez na adolescência e de violência doméstica na comunidade. A partir da dança, a equipe observa que há uma perspectiva diferente de vida para as meninas da comunidade.

[...] a questão da dança que inchiu muitas adolescentes [...], gravidez precoce sempre foi um problema em Machadinha [...] a dança salvou muitas meninas, naquela ocasião da gravidez precoce e da violência doméstica [...]. (B)

Outro forte hábito cultural dessa população é a utilização das ervas medicinais com finalidade terapêutica $^{8}$. Apesar de a equipe reconhecer e valorizar essa tradição, parece haver preocupação dos profissionais com a falta do conhecimento científico por parte do grupo, no que diz respeito à utilização dessas ervas, conforme constatado nos discursos seguintes:

Já faz parte da cultura deles a utilização dessas ervas [...]. (P)

[...] vai passando de pai para filho a função delas, mas eles não conhecem cientificamente cada uma delas [...]. (D)

Observa-se, a partir desses depoimentos, que apesar do esforço da equipe em reconhecer e preservar os hábitos culturais dessa população na prestação do cuidado, ainda é considerado relevante legitimar tais práticas numa parceria com o saber técnico. Assim, a equipe desenvolve atividades que possam propiciar a troca de informações ${ }^{5,6}$, conforme identificado nos relatos a seguir.

[...] não queremos que eles parem de utilizar essas ervas, mas associar à medicação que é importante [...]. (P)

[...] junto com a coordenadora de farmácia [...] pegamos algumas ervas que eram comuns da região, algumas plantas que eles usavam e fizemos vários chás [...] tentamos valorizar esses saberes [...] da farmácia fitoterápica que eles usavam e chegamos a explorar [...] com eles. (B)

[...] fazemos reunião específica [...] vamos falar sobre as ervas que vocês usam, chás que vocês fazem, para que serve? Como é que vocês descobriram isso? Isso foi passado pela sua mãe ou avó? Quem é que faz? Como que faz? E a gente vai valorizando dessa forma [...]. (D)

O diálogo entre os diversos conhecimentos contribui para o enfrentamento mais efetivo dos problemas de saúde da população, pois eles se complementam. Nesse sentido, perceber o que os diferentes grupos sociais compreendem sobre o processo saúde-doença e sobre os recursos terapêuticos disponíveis significa abrir um leque de possibilidades de cuidado ${ }^{6}$.

\section{$2^{a}$ Categoria: Estratégias da equipe de saúde da família para acompanhamento dos usuários}

Nos discursos selecionados, percebe-se que a equipe de saúde tem como estratégia abrir espaço nas reuniões comunitárias para que a comunidade sugira os temas a serem abordados nas atividades educativas $^{10}$, isto é, os assuntos são voltados para o real interesse e necessidade da população. Tal prática retrata uma perspectiva educativa dialogada e emancipatória, acolhendo a cultura e o saber popular ${ }^{5}$.

[...] a gente faz as reuniões comunitárias e as pessoas trazem os assuntos, e a gente vai valorizando dessa forma [...] é mais interessante falar de repente sobre bicho de pé que é [...] mais comum para eles, ou sobre o pano branco, como evitar... [...] eles trazem para gente as dúvidas [...]. (B)

Outra estratégia da equipe para promover a aproximação dos funcionários da ESF com os moradores da comunidade, a fim de construir parcerias e fortalecer o vínculo, foi um se inserir no cotidiano do outro, pois a mudança desejada dependia de um caminhar conjunto ${ }^{5,6}$.

Eu comecei a participar do time de futebol da comunidade [...] jogava bola toda semana com os homens... [...] O acolhimento é a forma mais prática de você conseguir fazer com que a pessoa entenda as dificuldades que ela pode ter em determinado assunto. (B)

O acolhimento é fundamental [...]. (P)

[...] Criticar e ter preconceito são as maneiras mais dificeis de você conseguir mudar, você tem que ter uma 
empatia pela pessoa, por aquele problema e tentar passar através de sua experiência ou técnicas, comparando com as experiências de outras pessoas [...] a gente tenta orientar, mas sempre respeitando a cultura deles [...]. (D)

Um fator que limitou o trabalho da equipe foi o alto índice de analfabetismo e semianalfabetismo presente na comunidade ${ }^{4}$. Com o intuito de facilitar a comunicação entre os trabalhadores de saúde e a população, a equipe buscou aproximações com o vocabulário do grupo, de modo a reconhecer a sua cultura para facilitar a interação e o diálogo 5-7.

[...] então a gente tentava sempre trazer para nossas discussões alguns termos que eles usavam, e a gente estava sempre discutindo [...]. (B)

[...] através do trabalho da unidade de saúde a gente vai debatendo sempre [...]. (M)

De acordo com a teoria transcultural do cuida$\mathrm{do}^{7}$, é imprescindível que os círculos de cultura estabelecidos entre profissionais e clientes se fortaleçam e sejam oriundos de diálogos verdadeiros e sistemáticos para que os usuários compreendam os códigos da área de saúde e, dessa forma, estejam empoderados para a tomada de decisão.

Comprovou-se que, de um modo geral, as práticas de saúde desenvolvidas pela equipe buscaram ampliar os canais de interação cultural, por meio dos quais os interesses e problemas da população foram compreendidos pelos profissionais como parte de um processo. Essa compreensão contribuiu, significativamente, para a possível identificação e resolução de problemas de saúde, considerando a interação entre o saber técnico e o saber popular, com vistas a construir a complementaridade entre estes saberes, a qual pode contribuir para um cuidado dialogado, humanizado, integral e emancipatório.

\section{ConClusão}

Esta investigação constatou que os hábitos culturais da população quilombola de Machadinha estão, ainda, fortemente presentes, sobretudo na culinária, na dança e no uso de ervas medicinais. $\mathrm{O}$ índice de analfabetismo é elevado e, em decorrência desses aspectos, a equipe de saúde da família precisa valorizar a sua prática considerando a cultura local como norteadora no processo de cuidar.

As práticas alimentares e o uso das ervas medicinais exigem dos profissionais da saúde da família uma adequação quanto ao vocabulário e ao conhecimento transferido de geração para geração. É preciso estabelecer um diálogo entre o saber popular trazido por esse grupo e o saber técnico trazido pela equipe.

Nesse sentido, os profissionais utilizam como principal estratégia a reunião comunitária, considerada espaço legítimo para a troca, inclusive, permitindo à população decidir sobre diversos aspectos do serviço.
Outra estratégia mencionada foi o envolvimento dos profissionais nas atividades proporcionadas pela própria população, na medida em que essa atitude aproxima e facilita o vínculo. Além disso, há valorização das reuniões de equipe como lugar de diálogo e de planejamento conjunto das ações, considerando os diversos aspectos que envolvem as questões culturais específicas do grupo.

Como limitações do estudo, destaca-se o número reduzido de pesquisas sobre a população quilombola e os aspectos que envolvem a relação da cultura com o cuidado prestado pela equipe de saúde. Além disso, houve participação incipiente dos sujeitos do estudo durante a realização do grupo focal, pois, apesar de possuírem grande vivência na comunidade, não se sentiram à vontade para darem sua contribuição, mesmo com o destaque feito quanto ao sigilo e confidencialidade do estudo.

\section{REFERÊNCIAS}

1.Langdon, EJ, Wiik FB. Antropologia, saúde e doença: uma introdução ao conceito de cultura aplicado às ciências da saúde. Rev Latino-Am Enfermagem. 2010; 18:459-66. 2.Furtado MB, Pedroza RLS, Alves CB. Cultura, identidade e subjetividade quilombola: uma leitura a partir da psicologia cultural. Psicol Soc. 2014; 26:106-15.

3.Ministério da Saúde (Br). Departamento de Atenção Básica. Política Nacional de Atenção Básica. Brasília (DF): Ministério da Saúde; 2012.

4.Ministério da Saúde $(\mathrm{Br})$. Departamento de Apoio à Gestão Participativa. Política Nacional de Saúde Integral da População Negra: uma política para o SUS. Brasília (DF): Ministério da Saúde; 2013.

5. David HMSL, Bonetti OP, Silva MRF. A enfermagem brasileira e a democratização da saúde: notas sobre a política nacional de educação popular em saúde. Rev Bras Enferm. 2012; 65:179-85.

6.Ricardo LM, Stotz EN. Educação popular como método de análise: relações entre medicina popular e a situação-limite vivenciada por trabalhadores do movimento dos trabalhadores rurais sem-terra. Rev APS. 2012; 15:435-42. 7.Leininger MM. Teoria do cuidado transcultural: diversidade e universalidade. 2012. [citado em 10 ago 2015] Disponível em: http//www.portal educação.com

8. Silva LV. Machadinha: origem, história e influência. Rio de Janeiro: Editora Gráfica Ltda; 2009.

9. Balbi A. Quissamã: a raiz de uma história. Rio de janeiro: Sol Gráfico Ltda; 2011.

10.Acioli S, David HML, Faria MGA. Educação em saúde e a enfermagem em saúde coletiva: reflexões sobre a prática. Rev enferm UERJ. 2012; 20:533-6.

11.Martins PAF, Alvim NAT. Plano de cuidados compartilhado: convergência da proposta educativa problematizadora com a teoria do cuidado cultural de enfermagem. Rev Bras Enferm. 2012; 65:368-73.

12. Bardin L. Análise de conteúdo. Tradução de Luis Antero Reto e Augusto Pinheiro. São Paulo: Edições 70; 2011. 